\title{
MULTIPLICITY OF SOLUTIONS FOR A FRACTIONAL $p$ - KIRCHHOFF TYPE PROBLEM WITH SIGN-CHANGING WEIGHTS FUNCTION
}

\section{YUYAN GUI}

Abstract. In this paper, we consider the existence of multiple solutions for the following fractional $p$ - Kirchhoff type problem

$$
\begin{cases}\left(\iint_{\mathbb{R}^{2 n}} \frac{|u(x)-u(y)|^{p}}{|x-y|^{n+p s}} d x d y\right)^{\theta / p}(-\Delta)_{p}^{s} u=f(x)|u|^{q-1} u+g(x)|u|^{r-1} u, & \text { in } \Omega, \\ u=0, & \text { in } \mathbb{R}^{n} \backslash \Omega,\end{cases}
$$

where $\Omega$ is an open bounded set in $\mathbb{R}^{n}, p>1, \theta \geqslant 0,0<q<\theta+p-1<r<p_{s}^{*}-1$ with $p_{s}^{*}=$ $\frac{n p}{n-p s}$ for $n>p s$ and $s \in(0,1)$ fixed, $f(x)$ and $g(x)$ are sign-changing continuous functions in $\Omega,(-\triangle)_{p}^{s} u$ denotes the fractional $p$-Laplacian operator. We obtain the multiplicity of solutions to (0.1) by using fibering map analysis and the Nehari manifold approach.

Mathematics subject classification (2010): 35J20, 35J92.

Keywords and phrases: Kirchhoff type problem, fractional $p$-Laplacian, fibering map analysis, Nehari manifold approach.

\section{REFERENCES}

[1] G. Autuori, A. Fiscella, P. PuCCI, Stationary Kirchhoff problems involving a fractional elliptic operator and a critical nonlinearity, Nonlinear Anal. 125, (2015), 699-714.

[2] G. Autuori, F. Colasuonno, P. PuCCI, On the existence of stationary solutions for higher-order p-Kirchhoff problems, Commun. Contemp. Math. 16, (2014), no. 5, 1450002, 43 pp.

[3] C. Brändle, E. Colorado, A. De Pablo, U. SÁnchez, A concave-convex elliptic problem involving the fractional Laplacian, Proc. Roy. Soc. Edinburgh Sect. A, 143, (01) (2013), 39-71.

[4] C. BAI, Multipicity results for a fractional Kirchhoff equation involving sign-chaging weight function, Bound. value probl. 2016, (1)2016, 1-16.

[5] C. M. CHU, J. J. SUn, Z. P. CAI, Multiple solutions for a Kirchhoff-type problem involving nonlocal fractional p-Laplacian and concave-convex nonlinearities, Rocky Mountain J.Math. 47,(2017), no. 6, 1803-1823.

[6] K. BRown, Y. ZhANG, The Nehari manifold for a semilinear elliptic equation with a sign-changing weight function, J. Diff. Equations, 193, (2003), 481-499.

[7] W. Chen, S. Deng, Existence of solution for a Kirchhoff type problem involving the fractional $p$-Laplace operator, Electron. J. Qual. Theory Differ. Equ., (2015), No. 87, 1-8.

[8] W. Chen, S. Deng, The Nehari manifold for a fractional p-Laplacian system involving concaveconvex nonlinearities, Nonlinear Anal. Real World Appl. 27,(2016), 80-92.

[9] W. CHEN, Y. GUI, Multiple solutions for a fractional p-Kirchhoff problem with Hardy nonlinearity, Nonlinear Analysis, 188, (2019), 316-338.

[10] W. Chen, M. Squassina, Critical Nonlocal Systems with Concave-Convex Powers, Advance Nonlinear Studies, 16, (4)(2016), 821-842.

[11] F. Colasuonno, P. PuCCI, Multiplicity of solutions for p $(x)$ - polyharmonic elliptic Kirchhoff equations, Nonlinear Anal. 74, (17)(2011), 5962-5974.

[12] E. Di NezZa, G. Palatucci, E. Valdinoci, Hitchhiker's guide to the fractional Sobolev spaces, Bull. Sci. math., 136, (2012), 521-573. 
[13] M. Dreher, The Kirchhoff equation for the p-Laplacian, Rend. Semin. Mat. (Torino), 64, (2006), 217-238.

[14] A. Fiscella, P. K. Mishra, The Nehari manifold for fractional Kirchhoff problems involving singular and critical terms, Nonlineae Anal. 186, (2019), 6-32.

[15] A. Fiscella, E. VAlDinOCi, A critical Kirchhoff type problem involving a nonlocal operator, Nonlinear Anal. 94, (2014), 156-170.

[16] M. Ferrara, B. Zhang, M. XIANG, Multiplicity results for the nonhomogeneous fractional pKirchhoff equations with concave-convex nonlinearities, Proc.R.Soc. A 471: 20150034.

[17] S. GoYAL, K. SREENADH, Nehari manifold for non-local elliptic operator with concave-convex nonlinearities and sign-changing weight functions, Proceedings Mathematical Sciences, 125, (4)(2015), $545-558$.

[18] S. Goyal, K. SREenAdh, Existence of multiple solutions of p-fractional Laplace operator with signchanging weight function, Adv. Nonlinear Anal. 4, (1)(2015), 37-58.

[19] A. OURRAOUI, On a p-Kirchhoff problem involving a critical nonlinearity, C. R. Acad. Sci. Paris, Ser. I, 352, (2014), 295-298.

[20] R. Lehrer, L. A. Maia, M. SQuassina, On fractional p-Laplacian problems with weight, Differential \& Integral Equations, 28, (2015), 15-28.

[21] J. LiAO, Y. PU, X. Ke, C. TANG, Multiple positive solutions for Kirchhoff type problems involving concave-convex nonlinearities, Commun. Pure Appl. Anal. 16, (2017), no. 6, 2157-2175.

[22] A. Iannizzotto, S. Liu, K. Perera, M. SQuassina, Existence results for fractional p-Laplacian problems via Morse theory, Adv. Calc. Var. 9, (2)(2016), 101-125.

[23] G. SUN, K. TENG, Existence and multiplicity of solutions for a class of fractional Kirchhoff-type problem, Math. Commun., 19, (2014), 183-194.

[24] T. F. WU, Multiplicity results for a semilinear elliptic equation involving sign-chaging weight function, Rocky Mountain J. Math. 39, (2009), no.3, 995-1011.

[25] Y. WeI, C. CHEN, H. Song, Multiplicity of solutions for a class of fractional p-Kirchhoff system with sgin-changing weight functions, Bound. Value Problem. 2018, (1)2018, 1-18.

[26] J. Zou, T. AN, L. REN, The Nehari manifold for a fractional p-Kirchhoff system involving sign-changing weight function and concave-convex nonlinrarities, J. Founct. Spaces 2019, Art. Id 7624373,9 . 